\title{
COMMENTARY ON AN UNPUBLISHED LECTURE BY G. N. WATSON ON SOLVING THE QUINTIC
}

\author{
by \\ Bruce C. Berndt \\ Department of Mathematics, University of Illinois \\ Urbana, Illinois, USA 61801 \\ Blair K. Spearman \\ Department of Mathematics and Statistics, Okanagan University College \\ Kelowna, British Columbia, Canada V1V 1V7 \\ Kenneth S. Williams \\ School of Mathematics and Statistics, Carleton University \\ Ottawa, Ontario, Canada K1S 5B6
}

The following notes are from a lecture on solving quintic equations given by the late Professor George Neville Watson (1886 - 1965) at Cambridge University in 1948. They were discovered by the first author in 1995 in one of two boxes of papers of Professor Watson stored in the Rare Book Room of the Library at the University of Birmingham, England. Some pages that had become separated from the notes were found by the third author in one of the boxes during a visit to Birmingham in 1999.

"Solving the quintic" is one of the few topics in mathematics which has been of enduring and widespread interest for centuries. The history of this subject is beautifully illustrated in the poster produced by MATHEMATICA. Many attempts have been made to solve quintic equations; see, for example, [6]-[14], [17]-[21], [28][32], [34]-[36], [58]-[60]. Galois was the first mathematician to determine which quintic polynomials have roots expressible in terms of radicals, and in 1991 Dummit [24] gave formulae for the roots of such solvable quintics. A quintic is solvable by means of radicals if and only if its Galois group is the cyclic group $\mathbb{Z}_{5}$ of order 5 ,

The first author thanks Professor Norrie Everitt of the University of Birmingham for an invitation to visit the University of Birmingham in October 1995.

The third author thanks Carleton University for a travel grant which enabled him to travel to the University of Birmingham, England in December 1999.

The authors thank the staff of the University of Birmingham Library for making the papers of Watson available to them. 


\section{AN UNPUBLISHED LECTURE OF WATSON ON SOLVING THE QUINTIC}

the dihedral group $D_{5}$ of order 10, or the Frobenius group $F_{20}$ of order 20 . In view of the current interest (both theoretical and computational) in solvable quintic equations [24], [33], [43]-[46], it seemed to the authors to be of interest to publish Professor Watson's notes on his lecture, with commentary explaining some of the ideas involved in more current mathematical language. For those having a practical need for solving quintic equations, Watson's step-by-step procedure will be especially valuable. Watson's method applies to any solvable quintic polynomial, that is, any quintic polynomial whose Galois group is one of $\mathbb{Z}_{5}, D_{5}$ or $F_{20}$.

Watson's interest in solving quintics was undoubtedly motivated by his keen interest in verifying Ramanujan's determinations of class invariants, or equivalently, singular moduli. Ramanujan computed the values of over 100 class invariants, which he recorded without proofs in his paper [37] and at scattered places throughout his notebooks, especially in his first notebook [39]. Although many of Ramanujan's class invariants had been also calculated by Weber [57], most had not been heretofore verified. Class invariants are certain algebraic numbers which are normally very difficult to explicitly calculate, and so their determinations often require solving a polynomial equation of degree greater than 2, and, in particular, 5. Watson [52] used modular equations in calculating some of Ramanujan's class invariants, with solving polynomial equations of degree exceeding 2 often needed. In a series of six further papers [50] - [51], [53] - [56], he developed an empirical process for calculating class invariants, which also heavily depended on solving polynomial equations of high degree. He not only verified several of Ramanujan's class invariants, but found many new ones as well. For these reasons, Watson proclaimed in his lecture that he had solved more quintic equations than any other person. Despite Watson's Gargantuan efforts in calculating Ramanujan's class invariants, eighteen remained unproven until recent times. The remaining ones were verified in two papers by Berndt, Chan and Zhang [2], [3]; see also Berndt's book [1, Part V, Chapter 34]. Chan [16] has used class field theory to put Watson's determinations on a firm foundation, and Zhang [61], [62] has used Kronecker's limit formula to also verify Watson's calculations.

Professor Watson held the Mason Chair of Mathematics at the University of Birmingham from 1918 to 1951. He was educated at Cambridge University (19041908), where he was a sudent of Edmund Taylor Whittaker (1873-1956). He became a Fellow of Trinity College, Cambridge in 1910. From 1914-1918 he held academic positions at University College, London. Watson devoted a great deal of his research to extending and providing proofs for results contained in Ramanujan's Notebooks [39]. He wrote more than thirty papers related to Ramanujan's work, including the aforementioned papers on class invariants, or singular moduli.

Most mathematicians know Watson as the co-author with E. T. Whittaker of the classic book, "A Course of Modern Analysis," first published in 1915, as well as the author of the monumental treatise, "Theory of Bessel Functions," first published in 
1922. For more details of Watson's life, the reader may wish to consult [22], [41], [48].

We now give the text of Watson's lecture with our commentary in italics. At the end of the text we give the contents of three sheets which presumably Watson handed out to his audience. The first of these gives the basic quantities associated with a quintic equation, the second gives twenty-four pentagrams used in showing that permutations of the suffixes of

$$
\left(x_{1} x_{2}+x_{2} x_{3}+x_{3} x_{4}+x_{4} x_{5}+x_{5} x_{1}-x_{1} x_{3}-x_{3} x_{5}-x_{5} x_{2}-x_{2} x_{4}-x_{4} x_{1}\right)^{2}
$$

yield six distinct expressions, and the third gives Watson's method of solving a solvable quintic equation in radicals.

I am going to begin by frankly admitting that my subject this evening is definitely old-fashioned and is rather stodgy; you will not find anything exciting or thrilling about it. When the subject of quintic equations was first seriously investigated by Lagrange it really was a "live" topic; the extent of the possibility of solving equations of various degrees by means of radicals was of general interest until it was realized that numbers represented by radicals and roots of algebraic equations were about what one nowadays calls algebraic numbers.

It is difficult to know quite how much to assume that you already know about solutions of algebraic equations but I am going to take for granted...

Watson's notes do not state the prerequisites for the lecture!

I cannot begin without saying how much I value the compliment which you have paid me by inviting me to come from a provincial University to lecture to you in Cambridge; and now I am going to claim an old man's privilege of indulging in a few reminiscences. In order to make my lecture effective, I must endeavour to picture to myself what is passing in the mind of John Brown who is sitting somewhere in the middle of this room and who came up to Trinity last October; and I suppose that, in view of the recent decision about women's membership of the University, with the name of John Brown I must couple the name of his cousin Mary Smith who came up to Newnham at the same time. To try to read their thoughts I must cast my mind back 43 years to the Lent Term of 1905 which was in my first year. If I had then attended a lecture by a mathematician 43 years my senior who was visiting Cambridge, an inspection of the Tripos lists would show that the most likely person to satisfy the requisite conditions would have been the late Lord Rayleigh, who was subsequently Chancellor. Probably to you he seems quite prehistoric; to me he was an elderly and venerable figure whose acquaintance I made in 1912, and with whom I subsequently had some correspondence about electric waves. You cannot 
help regarding me as equally elderly, but I hope that, for a number of reasons you do not consider me equally venerable, and that you will believe me when I say that I still have a good deal of the mentality of the undergraduate about me.

However, so far as I know, Lord Rayleigh did not visit Cambridge in the Lent term of 1905, and so my attempt at an analogy rather breaks down. On the other hand a visit was paid to Cambridge at the end of that term by a much more eminent personage, namely the Sultan of Zanzibar. For the benefit of those of you who have not heard that story, I mention briefly that on the last day of term the Mayor of Cambridge received a telegram to the effect that the Sultan and his suite would be arriving by the mid-day train from King's Cross and would be glad if the Mayor would give them lunch and arrange for them to be shown over Cambridge in the course of the afternoon. The program was duly carried out, and during the next few weeks it gradually emerged that the so-called Sultan was W.H. de Vere Cole, a third-year Trinity undergraduate. It was the most successful practical joke of an age in which practical joking was more popular than it is to-day.

If you could be transported back to the Cambridge of 1905, you would find that it was not so very different from the Cambridge of 1948. One of the differences which would strike you most would probably be the fact that there were very few University lectures (and those mostly professorial lectures which were not much attended by undergraduates); other lectures were College lectures, open only to members of the College in which they were given, or, in the case of some of the smaller Colleges, they were open to members of two or three colleges which had associated themselves for that purpose. Thus most of the teaching which I received was from the four members of the Trinity mathematical staff; the senior of them was Herman, who died prematurely twenty years ago, in addition to teaching me solid geometry, rigid dynamics and hydrodynamics, he infected me with a quality of perseverance and tenacity of purpose which I think was less uncommon in the nineteenth century than it is to-day when mathematics is tending to be less concrete and more abstract. Whitehead was still alive when I started collecting material for this lecture. Whittaker, who lectured on Electricity and Geometric Optics, whose name is sometimes associated with mine, is living in retirement in Edinburgh; and Barnes is Bishop of Birmingham. Outside the College I attended lectures by Baker on Theory of Functions, Berry of King's who taught me nearly all of what I know of elliptic functions, and Hobson on Spherical Harmonics and Integral Equations; also two of the Professors of that time that were Trinity men, Forsyth and Sir George Darwin whom I remember lecturing on curvature of surfaces and the problem of three bodies respectively. Two things you may have noticed, the large proportion of my teachers who are still alive, and the insularity, if I may so describe it, of my education. If that hypothetical lecture by Lord Rayleigh had taken place, he could have given a more striking illustration of insularity which you will probably hardly 
credit. In his time, each College tutor was responsible for the teaching of his own pupils and of nobody else; he was aided by one or two Assistant Tutors, but the pupils, no matter what subject they were reading, received no official instruction except from their own tutor and his assistants.

After spending something like ten minutes on these irrelevancies, it is time that I started getting to business.

There is one assumption which I am going to make throughout, namely that the extent of your knowledge about the elements of the theory of equations is roughly the same as might have been expected of a similar audience in 1905. For instance, I am going to take for granted that you know about symmetric functions of roots in terms of coefficients and that you are at any rate vaguely familiar with methods of obtaining algebraic solutions of quadratic, cubic and quartic equations, and that you have heard of the theorem due to Abel that there is no such solution of the general quintic equation, i.e. a solution expressible by a number of root extractions.

In modern language, if $f(x) \in \mathbb{Q}[x]$ is irreducible and of degree 5 then the quintic equation $f(x)=0$ is solvable by radicals if and only if the Galois group $G$ of $f(x)$ is solvable. The Galois group $G$ is solvable if and only if it is a subgroup of the Frobenius group $F_{20}$ of order 20, that is, it is $F_{20}, D_{5}$ (the dihedral group of order 10 ), or $\mathbb{Z}_{5}$ (the cyclic group of order 5); see for example [24, Theorem 2 , p. 397], [25, Theorem 39, p. 609]. Thus a quintic equation $f(x)=0$ cannot have its roots expressed by a finite number of root extractions if the Galois group $G$ of $f$ is non-solvable, that is, if it is $S_{5}$ (the symmetric group of order 120 ), or $A_{5}$ (the alternating group of order 60). "Amost all" quintics have $S_{5}$ as their Galois group, so the "general" quintic is not solvable by radicals. It is easy to give examples of quintics which are not solvable by radicals; see for example [46].

You may or may not have encountered the theorem that any irreducible quintic which has got an algebraic solution has its roots expressible in the form

$$
u_{0}+\omega^{r} u_{1}+\omega^{2 r} u_{2}+\omega^{3 r} u_{3}+\omega^{4 r} u_{4}
$$

where $\omega$ denotes $\exp (2 \pi i / 5), r$ assumes the values $0,1,2,3,4$, and $u_{1}^{5}, u_{2}^{5}, u_{3}^{5}, u_{4}^{5}$ are the roots of a quartic equation whose coefficients are rational functions of the coefficients of the original quintic. If you are not familiar with such results, you will find proofs of them in the treatise by Burnside and Panton.

One can find this in Section 5 of Chapter XX of Burnside and Panton's book [5, Vol. 2]. A modern reference for this result is [24, Theorem 2, p. 397]. 


\section{AN UNPUBLISHED LECTURE OF WATSON ON SOLVING THE QUINTIC}

When I was an undergraduate, all other knowledge about quintic equations was hidden behind what modern politicians would describe as an iron curtain, and it is convenient for me to assume that this state of affairs still persists, for otherwise it would be a work of supererogation for me to deliver this lecture.

I might mention at this point that equations of the fifth or a higher degree which possess algebraic solutions (such equations are usually described as Abelian) are of some importance in the theory of elliptic functions, apart from their intrinsic interest.

Today such equations are called solvable.

There is, for instance, a theorem, also due to Abel, that the equations satisfied by the so-called "singular moduli" of elliptic functions are all Abelian equations.

Singular moduli are discussed in Cox's book [23, Chapter 3] as well as in Berndt's book [1, Part V, Chapter 34].

It was these singular moduli which aroused my interest some fifteen years ago in the solutions of Abelian equations, not only of the fifth degree, but also of the sixth, seventh and other degrees higher still. It consequently became necessary for me to co-ordinate the work of previous writers in such a way as to have handy a systematic procedure for solving Abelian quintic equations as rapidly as possible, and this is what I am going to describe tonight.

Methods for solving a general solvable quintic equation in radicals have been given in the 1990's by Dummit [24] and Kobayashi and Nakagawa [33]; see also [47].

To illustrate the nature of the problem to be solved, I am now going to use equations of degrees lower than the fifth as illustrations. A reason why such equations possess algebraic solutions (and it proves to be the reason) is that certain non-symmetric functions of the roots exist such that the values which certain powers of them can assume are fewer in number than the degree of the equation. Thus, in the case of the quadratic equation with roots $\alpha$ and $\beta$, there are two values for the difference of the roots, namely

$$
\alpha-\beta, \beta-\alpha
$$

However the squares of both of these differences have one value only, namely

$$
(\alpha+\beta)^{2}-4 \alpha \beta
$$


and this is expressible rationally in terms of the coefficients. Hence the values of the differences of the roots are obtainable by the extraction of a square root, and, since the sum of the roots is known, the roots themselves are immediately obtainable.

The cubic equation, with roots $\alpha, \beta, \gamma$, can be treated similarly. Let $\epsilon^{3}=1$ $(\epsilon \neq 1)$. Then we can form six expressions

$$
\begin{array}{lll}
\alpha+\beta \epsilon+\gamma \epsilon^{2}, & \beta+\gamma \epsilon+\alpha \epsilon^{2}, & \gamma+\alpha \epsilon+\beta \epsilon^{2}, \\
\alpha+\beta \epsilon^{2}+\gamma \epsilon, & \beta+\gamma \epsilon^{2}+\alpha \epsilon, & \gamma+\alpha \epsilon^{2}+\beta \epsilon,
\end{array}
$$

with the property that their cubes have, not six different values, but only two, namely

$$
\left(\alpha+\beta \epsilon+\gamma \epsilon^{2}\right)^{3}, \quad\left(\alpha+\beta \epsilon^{2}+\gamma \epsilon\right)^{3}
$$

and these expressions are the roots of a quadratic equation whose coefficients are rational functions of the coefficients of the cubic. When the cubic equation is

$$
a x^{3}+3 b x^{2}+3 c x+d=0
$$

the quadratic equation is

$$
a^{6} X^{2}+27 a^{3}\left(a^{2} d-3 a b c+2 b^{3}\right) X+729\left(b^{2}-a c\right)^{3}=0
$$

and there is no difficulty in completing the solution of the cubic.

It is easily checked using MAPLE that this quadratic is correct.

For the quartic equation, with roots $\alpha, \beta, \gamma, \delta$, such expressions as

$$
(\alpha+\beta-\gamma-\gamma)^{2}, \quad(\alpha+\gamma-\delta-\beta)^{2}, \quad(\alpha+\delta-\beta-\gamma)^{2}
$$

have only three distinct values; similar but slightly simpler expressions are

$$
\alpha \beta+\gamma \delta-\alpha \gamma-\alpha \delta-\beta \gamma-\beta \delta, \text { etc. }
$$

or simpler still,

$$
\alpha \beta+\gamma \delta, \quad \alpha \gamma+\beta \delta, \quad \alpha \delta+\beta \gamma
$$

When the quartic equation is taken to be

$$
a x^{4}+4 b x^{3}+6 c x^{2}+4 d x+e=0,
$$

the cubic equation satisfied by the last three expressions is

$$
a X^{3}-6 a^{2} c X^{2}+(16 b d-4 a e) a X-\left(16 b^{2} e+16 a d^{2}-24 a c e\right)=0
$$


and, by the substitution

$$
a X-2 c=-4 \theta
$$

this becomes

$$
4 \theta^{3}-\theta\left(a e-4 b d+3 c^{2}\right)-\left(a c e+2 b c d-a d^{2}-b^{2} e-c^{3}\right)=0
$$

which is the standard reducing cubic

$$
4 \theta^{3}-I \theta-J=0
$$

This is discussed in [26, pp. 191-197; see problem 15, p. 197 ], where the values of $I$ and $J$ are given by

$$
I=a e-4 b d+3 c^{2}, \quad J=\left|\begin{array}{lll}
a & b & c \\
b & c & d \\
c & d & e
\end{array}\right|
$$

I have discussed the problem of solving the quartic equation at some length in order to be able to point out to you the existence of a special type of quartic equation which rarely receives the attention that it merits. In general the reducing cubic of a quartic equation has no root which is rational in the field of its coefficients, and any expression for the roots of the quartic involves cube roots; on the other hand, there is no difficulty in constructing quartic equations whose reducing cubics possess at least one rational root; the roots of such quartics are obtainable in forms which involve the extraction of square roots only. Such quartics are analogous to Abelian equations of higher degrees, and it might be worth while to describe them either as "Abelian quartic equations" or as "biquadratic equations," the latter being an alternative to the present usage of employing the terms quartic and biquadratic indifferently. (I once discussed this question with my friend Professor Berwick, who in his lifetime was the leading authority in this country on algebraic equations, and we both rather reluctantly came to the conclusion that the existing terminology was fixed sufficiently firmly to make any alteration in it practically impossible.)

If $f(x) \in \mathbb{Q}[x]$ is an irreducible quartic polynomial, its cubic resolvent has at least one rational root if and only if the Galois group of $f(x)$ is the Klein 4-group $V_{4}$ of order 4 , the cyclic group $\mathbb{Z}_{4}$ of order 4 , or the dihedral group $D_{4}$ of order 8. Since $D_{4}$ is not abelian, it is not appropriate to call such quartics "abelian." For the solution of the quartic by radicals, see for example [25, p. 548].

After this very lengthy preamble, I now reach the main topic of my discourse, namely quintic equations. Some of you may be familiar with the name of William 
Hepworth Thompson, who was Regius Professor of Greek from 1853 to 1866, and subsequently Master of Trinity until 1886. A question was once put to him about Greek mathematics, and his reply was "I know nothing about the subject. I have never even lectured upon it." Although there are large tracts of knowledge about quintic equations about which I am in complete ignorance, I have a fair amount of practical experience of them. For instance, if my friend Mr. P. Hall of King's College is here this evening, he will probably be horrified at the ignorance which I shall show when I say anything derived from the theory of groups. On the other hand, while to the best of my knowledge nobody else has solved more than about twenty Abelian quintics (you will be hearing later about these solvers, and I have no certain knowledge that anybody else has ever solved any), my own score is something between 100 and 120; and I must admit that I feel a certain amount of pride at having so far outdistanced my nearest rival.

Young solved several quintic equations in [58] and [59].

The notation which I use is given at the top of the first of the sheets which have been distributed. The first equation, namely

$$
p_{0} y^{5}+p_{1} y^{4}+p_{2} y^{3}+p_{3} y^{2}+p_{4} y+p_{5}=0,
$$

is what Cayley calls the denumerate form, while

$$
a x^{5}+5 b x^{4}+10 c x^{3}+10 d x^{2}+5 e x+f=0,
$$

is the standard form. The second is derived from the first by the substitution $10 y=x$, with the relations

$$
a=p_{0}, \quad b=2 p_{1}, \quad c=10 p_{2}, \quad d=100 p_{3}, \quad e=2000 p_{4}, \quad f=10^{5} p_{5} .
$$

Next we carry out the process usually described as "removing the second term" by the substitution $a x+b=z$, which yields the reduced form

$$
z^{5}+10 C z^{3}+10 D z^{2}+5 E z+F=0,
$$

in which

$$
\begin{aligned}
& C=a c-b^{2}, \quad D=a^{2} d-3 a b c+2 b^{3} \\
& E=a^{3} e-4 a^{2} b d+6 a b^{2} c-3 b^{4} \\
& F=a^{4} f-5 a^{3} b e+10 a^{2} b^{2} d-10 a b^{3} c+4 b^{5} .
\end{aligned}
$$

The roots of the last two quintics will be denoted by $x_{r}$ and $z_{r}$ respectively with $r=1,2,3,4,5$. 
10 AN UNPUBLISHED LECTURE OF WATSON ON SOLVING THE QUINTIC

Our next object is the determination of non-symmetric functions of the roots which can be regarded as roots of a resolvent equation. An expression which suggests itself is

$$
\left(x_{1}+\omega x_{2}+\omega^{2} x_{3}+\omega^{3} x_{4}+\omega^{4} x_{5}\right)^{5} .
$$

The result of permuting the roots is to yield 24 values for the expression.

A permutation $\sigma \in S_{5}$ acts on this element by

$$
\begin{aligned}
\sigma\left(\left(x_{1}+\omega x_{2}\right.\right. & \left.\left.+\omega^{2} x_{3}+\omega^{3} x_{4}+\omega^{4} x_{5}\right)^{5}\right) \\
& =\left(x_{\sigma(1)}+\omega x_{\sigma(2)}+\omega^{2} x_{\sigma(3)}+\omega^{3} x_{\sigma(4)}+\omega^{4} x_{\sigma(5)}\right)^{5} .
\end{aligned}
$$

An easy calculation shows that $\sigma$ preserves

$$
\alpha=\left(x_{1}+\omega x_{2}+\omega^{2} x_{3}+\omega^{3} x_{4}+\omega^{4} x_{5}\right)^{5}
$$

if and only if $\sigma=(12345)^{k}$ for some $k \in\{0,1,2,3,4\}$. Hence

$$
\operatorname{stab}_{S_{5}}(\alpha)=\langle(12345)\rangle,
$$

so that

$$
\left|\operatorname{stab}_{S_{5}}(\alpha)\right|=5 \text {. }
$$

Thus, by the orbit-stabilizer theorem [27, p. 139], we obtain

$$
\left|\operatorname{orb}_{S_{5}}(\alpha)\right|=\frac{\left|S_{5}\right|}{5}=\frac{120}{5}=24
$$

so that permuting the roots yields 24 different expressions.

The disadvantage of the corresponding resolvent equation is the magnitude of the degree of its coefficients when expressed as functions of the coefficients of the quintic; moreover it is difficult to be greatly attracted by an equation whose degree is as high as 24 when our aim is the solution of an equation of degree as low as 5 .

An expression which is more amenable than the expression just considered was discovered just 90 years ago by two mathematicians of some eminence in their day, namely Cockle and Harley, and it was published in the Memoirs of the Manchester Literary and Philosophical Society. This expression is

$$
\phi_{1}=x_{1} x_{2}+x_{2} x_{3}+x_{3} x_{4}+x_{4} x_{5}+x_{5} x_{1}-x_{1} x_{3}-x_{3} x_{5}-x_{5} x_{2}-x_{2} x_{4}-x_{4} x_{1} .
$$

The quantities $x_{1} x_{2}+x_{2} x_{3}+x_{3} x_{4}+x_{4} x_{5}+x_{5} x_{1}$ and $x_{1} x_{3}+x_{3} x_{5}+x_{5} x_{2}+x_{2} x_{4}+$ $x_{4} x_{1}$ appear in the work of Harley [29] and their difference is considered by Cayley 
[6]. We have not located a joint paper of Cockle and Harley. When writing these notes, we believe Watson was reading from Cayley [6] where the names of Cockle and Harley are linked [6, p. 311].

Permutations of the suffixes give rise to 24 expressions, which may be denoted by $\Sigma \pm x_{r} x_{s}$, where $r$ and $s$ run through the values $1,2,3,4,5$ with $r \neq s$. The choice of the signs is most simply exhibited diagrammatically, with each of the 24 expressions represented by a separate diagram. If you turn to the second page of your sheets, you will see the 24 pentagrams with vertices numbered $1,2,3,4,5$ in all possible orders (there is no loss of generality in taking the number 1 in a special place) and the rule for determination of signs is that terms associated with adjacent vertices are assigned + signs, while those associated with opposite vertices are assigned signs.

Now the pentagrams in the third and fourth columns are the optical images in a vertical line of the corresponding pentagrams in the first and second columns, and since proximity and oppositeness are invariant for the operation of taking an optical image, the number of distinct values of $\phi$ is reduced from 24 to 12 .

Further, the pentagrams in the second column are derived from those in the first column by changing adjacent vertices into opposite vertices, and vice versa, so that the values of $\phi$ arising from pentagrams in the second column are minus the values of $\phi$ arising from the corresponding pentagrams in the first column. It follows that the number of distinct values of $\phi^{2}$ is not 12 but 6 , and so our resolvent has now been reduced to a sextic equation in $\phi^{2}$, with coefficients which are rational functions of the coefficients of the quintic, and a sextic equation is a decided improvement on an equation of degree 120 , or even on one of degree 24 .

Let $a=(12345) \in S_{5}$ and $b=(25)(34) \in S_{5}$, so that $a^{5}=b^{2}=e$ and $b a b=a^{4}$. As $a \phi_{1}=\phi_{1}$ and $b \phi_{1}=\phi_{1}$, we have

$$
\operatorname{stab}_{S_{5}}\left(\phi_{1}\right) \geq\left\langle a, b \mid a^{5}=b^{2}=e, b a b=a^{4}\right\rangle=D_{5},
$$

so that

$$
\left|\operatorname{stab}_{S_{5}}\left(\phi_{1}\right)\right| \geq 10 \text {. }
$$

On the other hand, the first two columns of Watson's pentagram table show that

$$
\left|\operatorname{orb}_{S_{5}}\left(\phi_{1}\right)\right| \geq 12 \text {. }
$$

Hence, by the orbit-stabilizer theorem, we see that

$$
\left|\operatorname{stab}_{S_{5}}\left(\phi_{1}\right)\right|=10, \quad\left|\operatorname{orb}_{S_{5}}\left(\phi_{1}\right)\right|=12
$$


12 AN UNPUBLISHED LECTURE OF WATSON ON SOLVING THE QUINTIC

and thus

$$
\operatorname{stab}_{S_{5}}\left(\phi_{1}\right)=\left\langle a, b \mid a^{5}=b^{2}=e, b a b=a^{4}\right\rangle=D_{5} .
$$

Now let $c=(2345)$, so that $c^{2}=b$. As $a \phi_{1}^{2}=\phi_{1}^{2}$ and $c \phi_{1}^{2}=\phi_{1}^{2}$, we have

$$
\operatorname{stab}_{S_{5}}\left(\phi_{1}^{2}\right) \geq\left\langle a, c \mid a^{5}=c^{2}=e, c^{-1} a c=a^{3}\right\rangle=F_{20},
$$

so that $\left|\operatorname{stab}_{S_{5}}\left(\phi_{1}^{2}\right)\right| \geq 20$. From the first column of the pentagram table, we have

$$
\left|\operatorname{orb}_{S_{5}}\left(\phi_{1}^{2}\right)\right| \geq 6
$$

Hence, by the orbit-stabilizer theorem, we deduce that

$$
\left|\operatorname{stab}_{S_{5}}\left(\phi_{1}^{2}\right)\right|=20, \quad\left|\operatorname{orb}_{S_{5}}\left(\phi_{1}^{2}\right)\right|=6,
$$

and thus

$$
\operatorname{stab}_{S_{5}}\left(\phi_{1}^{2}\right)=\left\langle a, c \mid a^{5}=c^{4}=e, c^{-1} a c=a^{3}\right\rangle=F_{20} .
$$

It is, however, possible to effect a further simplification; it is not, in general, possible to construct a resolvent equation of degree less than 6 , but it is possible to construct a sextic resolvent equation in which two of the coefficients are zero. We succeeded in constructing a sextic in $\phi^{2}$ because the 12 values of $\phi$ could be grouped in pairs with the members of each pair numerically equal but opposite in sign; but a different grouping is also possible, namely a selection of one member from each of the six pairs so as to form a sestet in which the sum of the members is zero, and it is evident that those members which have not been selected also form a sestet in which the sum of the members is zero; one of these sestets is represented by the pentagrams in the first column, the other by the pentagrams in the second column.

A sestet is a set of six objects.

Denote the values of $\phi$ represented by the pentagrams in the first column by $\phi_{1}$, $\phi_{2}, \ldots, \phi_{6}$, and let

$$
\phi_{1}^{r}+\phi_{2}^{r}+\cdots+\phi_{6}^{r}=S_{r} .
$$

It is then not difficult to verify that an interchange of any pair of $x_{1}, x_{2}, \ldots, x_{5}$ changes the sign of $S_{r}$ when $r$ is odd, but leaves it unaltered in value when $r$ is even.

By looking at the first column of the pentagram table we see that the even permutations (234), (243), (354), (235), (24)(35) send $\phi_{1}$ to $\phi_{2}, \phi_{3}, \phi_{4}, \phi_{5}, \phi_{6}$, respectively. We next show that an odd permutation $\sigma$ cannot send $\phi_{i}$ to $\phi_{j}$ for any $i$ and $j$. 
Suppose that $\sigma\left(\phi_{i}\right)=\phi_{j}$. By the above remarks $\phi_{i}=\theta \phi_{1}$ for some $\theta \in A_{5}$, and $\phi_{1}=\rho \phi_{j}$ for some $\rho \in A_{5}$. Hence

$$
(\rho \sigma \theta) \phi_{1}=(\rho \sigma) \phi_{i}=\rho \phi_{j}=\phi_{1}
$$

so that

$$
\rho \sigma \theta \in \operatorname{stab}_{S_{5}} \phi_{1}=D_{5} \subseteq A_{5} .
$$

Hence $\sigma \in A_{5}$, which is a contradiction. Now

$$
\left\{\sigma\left(\phi_{1}\right), \ldots, \sigma\left(\phi_{6}\right)\right\} \subseteq \operatorname{orb}_{S_{5}} \phi_{1}, \quad\left|\operatorname{orb}_{S_{5}} \phi_{1}\right|=12,
$$

and

$$
\left\{\sigma\left(\phi_{1}\right), \ldots, \sigma\left(\phi_{6}\right)\right\} \cap\left\{\phi_{1}, \ldots, \phi_{6}\right\}=\phi
$$

so that

$$
\left\{\sigma\left(\phi_{1}\right), \ldots, \sigma\left(\phi_{6}\right)\right\}=\left\{-\phi_{1}, \ldots,-\phi_{6}\right\} .
$$

Thus if $\tau \in S_{5}$ is a transposition,

$$
\tau\left(S_{r}\right)=\tau\left(\phi_{1}^{r}+\cdots+\phi_{6}^{r}\right)=\left(-\phi_{1}\right)^{r}+\cdots+\left(-\phi_{6}\right)^{r}=(-1)^{r} S_{r} .
$$

It is now evident that each of the 10 expressions $x_{m}-x_{n}(m, n=1,2,3,4,5 ; m<n)$ is a factor of $S_{r}$ whenever $r$ is an odd integer.

Clearly $S_{r} \in \mathbb{Z}\left[x_{1}, \ldots, x_{5}\right]$ and so can be regarded as a polynomial in $x_{1}$ with coefficients in $\mathbb{Z}\left[x_{2}, \ldots, x_{5}\right]$. Dividing $S_{r}$ by $x_{1}-x_{2}$, we obtain

$$
S_{r}=\left(x_{1}-x_{2}\right) q\left(x_{2}, \ldots, x_{5}\right)+r\left(x_{2}, \ldots, x_{5}\right),
$$

where

$$
q\left(x_{2}, \ldots, x_{5}\right), \quad r\left(x_{2}, \ldots, x_{5}\right) \in \mathbb{Z}\left[x_{2}, \ldots, x_{5}\right] .
$$

If $r$ is odd, the transposition (12) changes the above equation to

$$
-S_{r}=\left(x_{2}-x_{1}\right) q\left(x_{1}, x_{3}, x_{4}, x_{5}\right)+r\left(x_{1}, x_{3}, x_{4}, x_{5}\right) .
$$

Adding these two equations, we obtain

$0=\left(x_{1}-x_{2}\right)\left(q\left(x_{2}, x_{3}, x_{4}, x_{5}\right)-q\left(x_{1}, x_{3}, x_{4}, x_{5}\right)\right)+r\left(x_{2}, x_{3}, x_{4}, x_{5}\right)+r\left(x_{1}, x_{3}, x_{4}, x_{5}\right)$.

Taking $x_{1}=x_{2}$ we deduce that

$$
r\left(x_{2}, x_{3}, x_{4}, x_{5}\right)=0
$$


14 AN UNPUBLISHED LECTURE OF WATSON ON SOLVING THE QUINTIC

Hence

$$
S_{r}=\left(x_{1}-x_{2}\right) q\left(x_{2}, \ldots, x_{5}\right) .
$$

Thus $x_{1}-x_{2}$ divides $S_{r}$. Similarly $x_{m}-x_{n}$ divides $S_{r}$ for $m, n=1,2,3,4,5$, $m<n$. Hence

$$
\prod_{1 \leq m<n \leq 5}\left(x_{m}-x_{n}\right)
$$

divides $S_{r}$ when $r$ is an odd integer.

Now the degrees of $S_{1}$ and $S_{3}$ in the $x$ 's are respectively 2 and 6 , and so, since these numbers are less than 10, both $S_{1}$ and $S_{3}$ must be identically zero, while $S_{5}$ must be a constant multiple of

$$
\left(x_{1}-x_{2}\right)\left(x_{1}-x_{3}\right) \cdots\left(x_{3}-x_{5}\right)\left(x_{4}-x_{5}\right) .
$$

On the other hand, $S_{2}, S_{4}$, and $S_{6}$ are symmetric functions of the $x$ 's, and are consequently expressible as rational functions of the coefficients in the standard form of the quintic.

These properties ensure that the polynomial

$$
\left(\phi-\phi_{1}\right)\left(\phi-\phi_{2}\right) \cdots\left(\phi-\phi_{6}\right)
$$

has coefficients in $\mathbb{Q}$ or $\mathbb{Q}(\sqrt{D})$, where $D$ is the discriminant of the quintic, with the coefficients of $\phi^{5}$ and $\phi^{3}$ equal to zero.

Apart from the graphical representation by pentagrams (which, as the White Knight would say, is my own invention), all of the analysis which I have just been describing was familiar to Cayley in 1861; and he thereupon set about the construction of the sextic resolvent whose roots are $\phi_{1}, \phi_{2}, \ldots, \phi_{6}$. The result which he obtained was the equation

$$
a^{6} \phi^{6}-100 K a^{4} \phi^{4}+2000 L a^{2} \phi^{2}-800 a^{2} \phi \sqrt{5 \Delta}+40000 M=0
$$

in which the values of $K, L, M$ in terms of the coefficients of the quintic are those given on the first sheet, while $\Delta$ is the discriminant of the quintic in its standard form, that is to say, it is the product of the squared differences of the roots of the quintic multiplied by $a^{8} / 3125$. Its value, in terms of the coefficients occupies the lower half of the first sheet. 
The work of Cayley to which Watson refers is contained in [6], where on pages 313 and 314 Cayley introduces the pentagrams described by Watson. Note that the usual discriminant $D$ of the quintic is [26, p. 205]

$$
a^{8}\left(x_{1}-x_{2}\right)^{2}\left(x_{1}-x_{3}\right)^{2} \cdots\left(x_{4}-x_{5}\right)^{2}=3125 \Delta=5^{5} \Delta .
$$

There is no obvious way of constructing any simpler resolvent and so it is only natural to ask "Where do we go from here?" It seems fruitless to attempt to obtain an algebraic solution of the general sextic equation; for, if we could solve the general sextic equation algebraically, we could solve the general quintic equation by the insertion of a factor of the first degree, so as to convert it into a sextic equation. In this connection I may mention rather a feeble joke which was once perpetrated by Ramanujan. He sent to the Journal of the Indian Mathematical Society as a problem for solution:

Prove that the roots of the equation

$$
x^{6}-x^{3}+x^{2}+2 x-1=0
$$

can be expressed in terms of radicals.

This problem is the first part of Question 699 in [38]. It can be found in [40, p. 331]. A solution was given by Watson in [49]. It seems inappropriate to refer to this problem as a "feeble joke."

Some years later I received rather a pathetic letter from a mathematician, who was anxious to produce something worth publication, saying that he had noticed that $x+1$ was a factor of the expression on the left, and that he wanted to reduce the equation still further, but did not see how to do so. My reply to his letter was that the quintic equation

$$
x^{5}-x^{4}+x^{3}-2 x^{2}+3 x-1=0
$$

was satisfied by the standard singular modulus associated with the elliptic functions for which the period $i K^{\prime} / K$ was equal to $\sqrt{-79}$, and consequently it was an Abelian quintic, and therefore it could be solved by radicals; and I told him where he would find the solution in print. I do not know why Ramanujan inserted the factor $x+1$; it may have been an attempt at frivolity, or it may have been a desire to propose an equation in which the coefficients were as small as possible, or it may have been a combination of the two. 
16 AN UNPUBLISHED LECTURE OF WATSON ON SOLVING THE QUINTIC

On pages 263 and 300 in his second notebook [39], Ramanujan indicates that $2^{1 / 4} G_{79}$ is a root of the quintic equation $x^{5}-x^{4}+x^{3}-2 x^{2}+3 x-1=0$, see $[1$, Part V, p. 193]. For a positive integer $n$, Ramanujan defined $G_{n}$ by

$$
G_{n}=2^{-1 / 4} f(\sqrt{-n}),
$$

where, for any $z=x+i y \in \mathbb{C}$ with $y>0$, Weber's class invariant $f(z)[57$, Vol. 3 , p. 114] is defined in terms of the Dedekind eta function

$$
\eta(z)=e^{\pi i z / 12} \prod_{m=1}^{\infty}\left(1-e^{2 \pi i m z}\right)
$$

by

$$
f(z)=\frac{e^{-\pi i / 24} \eta\left(\frac{z+1}{2}\right)}{\eta(z)} .
$$

A result equivalent to Ramanujan's assertion was first proved by Russell [42] and later by Watson [53]; see also [54]. The solution of this quintic in radicals is given in [49]. In [38], Ramanujan also posed the problem of finding the roots of another sextic polynomial which factors into $x-1$ and a quintic satisfied by $G_{47}$. For additional comments and references about this problem, see [4] and [40, pp. 400-401]. Both Weber and Ramanujan calculated over 100 class invariants, but for different reasons. Class invariants generate Hilbert class fields, one of Weber's primary interests. Ramanujan used class invariants to explicitly calculate certain continued fractions and products of theta functions.

After this digression, let us return to the sextic resolvent; it is the key to the solution of the quintic in terms of radicals, provided that such a key exists. It is possible, by accident as it were, for the sextic resolvent to have a solution for which $\phi^{2}$ is rational, and the corresponding value of $\phi$ is of the form $\rho \sqrt{5 \Delta}$, where $\rho$ is rational. A knowledge of such a value of $\phi$ proves to be sufficient to enable us to express all the roots of the quintic in terms of radicals. In fact, when this happy accident occurs, the quintic is Abelian, and when it does not occur, the quintic is not Abelian.

If $\phi^{2} \in \mathbb{Q}$ it is clear from the resolvent sextic that $\phi=\rho \sqrt{5 \Delta}$ for some $\rho \in \mathbb{Q}$. We are not aware of any rigorous direct proof of the equivalence of $\phi^{2} \in \mathbb{Q}$ to the original quintic being solvable.

This is as far as Cayley went; he was presumably not interested in the somewhat laborious task of completing the details of the solution of the quintic after the determination of a root of his sextic resolvent. 
The details of the solution of an Abelian quintic were worked out nearly a quarter of a century later by a contemporary of Cayley, namely George Paxton Young. I shall not describe Young as a mathematician whose name has been almost forgotten, because he was not in fact a professional mathematician at all. The few details of his career that are known to me are to be found in Poggendorf's biographies of authors of scientific papers. He was born in 1819, graduated M.A. at Edinburgh, and was subsequently Professor of Logic and Metaphysics at Knox College, Toronto; he was also an Inspector of Schools, and subsequently Professor of Logic, Metaphysics and Ethics in the University of Toronto. He died at Toronto on February 26, 1889. His life was thus almost coextensive with Cayley's (born August 16, 1821, died January 26,1895 ). Young in the last decade of his life (and not until then) published a number of papers on the algebraic solution of equations, including three in the American Journal of Mathematics which among them contain his method of solving Abelian quintics.

These are papers [58], [59] and [60].

In style, his papers are the very antithesis of Cayley's. While Cayley could not (or at any rate frequently did not) write grammatical English, he always wrote with extreme clarity, and, when one reads his papers, one cannot fail to be impressed by the terseness and lucidity of his style, by the mastery which he exercises over his symbols, and by the feeling which he succeeds in conveying that, although he may have frequently suppressed details of calculation, the reader would experience no real difficulty in filling in the lacunae, even though such a task might require a good deal of labour.

On the other hand, when one is reading Young's work, it is difficult to decide what his aims are until one has reached the end of his work, and then one has to return to the beginning and read it again in the light of what one has discovered; his choice of symbols is often unfortunate; in fact when I am reading his papers, I find it necessary to make out two lists of the symbols that he is using, one list of knowns and the other of unknowns; finally, his results seemed to be obtained by a sheer piece of good fortune, and not as a consequence of deliberate and systematic strategy. A comparison of the writings of Cayley and Young shows a striking contrast between the competent draughtsmanship of the lawyer and pure mathematician on the one hand and the obscurity of the philosopher on the other.

The rest of my lecture I propose to devote to an account of a practical method of solving Abelian quintic equations. The method is in substance the method given by Young, but I hope that I have succeeded in setting it out in a more intelligible, systematic and symmetrical manner. 
18 AN UNPUBLISHED LECTURE OF WATSON ON SOLVING THE QUINTIC

Take the reduced form of the quintic equation

$$
z^{5}+10 C z^{3}+10 D z^{2}+5 E z+F=0,
$$

and suppose that its roots are

$$
z_{r+1}=\omega^{r} u_{1}+\omega^{2 r} u_{2}+\omega^{3 r} u_{3}+\omega^{4 r} u_{4}
$$

where

$$
\omega=\exp (2 \pi i / 5), \quad r=1,2,3,4,0 .
$$

Straightforward but somewhat tedious multiplication shows that the quintic equation with these roots is

$$
\begin{aligned}
z^{5}- & 5 z^{3}\left(u_{1} u_{4}+u_{2} u_{3}\right)-5 z^{2}\left(u_{1}^{2} u_{3}+u_{2}^{2} u_{1}+u_{3}^{2} u_{4}+u_{4}^{2} u_{2}\right) \\
& +5 z\left(u_{1}^{2} u_{4}^{2}+u_{2}^{2} u_{3}^{2}-u_{1} u_{2} u_{3} u_{4}-u_{1}^{3} u_{2}-u_{2}^{3} u_{4}-u_{3}^{3} u_{1}-u_{4}^{3} u_{3}\right) \\
& -\left(u_{1}^{5}+u_{2}^{5}+u_{3}^{5}+u_{4}^{5}-5 u_{1}^{3} u_{3} u_{4}-5 u_{2}^{3} u_{1} u_{3}-5 u_{3}^{3} u_{4} u_{2}-5 u_{4}^{3} u_{2} u_{1}\right. \\
& \left.+5 u_{1} u_{3}^{2} u_{4}^{2}+5 u_{2} u_{1}^{2} u_{3}^{2}+5 u_{3} u_{4}^{2} u_{2}^{2}+5 u_{4} u_{2}^{2} u_{1}^{2}\right)=0
\end{aligned}
$$

and a comparison of these two forms of the quintic yields four equations from which $u_{1}, u_{2}, u_{3}, u_{4}$ are to be determined, namely

$$
\begin{gathered}
u_{1} u_{4}+u_{2} u_{3}=-2 C, \\
u_{1}^{2} u_{3}+u_{2}^{2} u_{1}+u_{3}^{2} u_{4}+u_{4}^{2} u_{2}=-2 D, \\
u_{1}^{2} u_{4}^{2}+u_{2}^{2} u_{3}^{2}-u_{1} u_{2} u_{3} u_{4}-u_{1}^{3} u_{2}-u_{2}^{3} u_{4}-u_{3}^{3} u_{1}-u_{4}^{3} u_{3}=E, \\
u_{1}^{5}+u_{2}^{5}+u_{3}^{5}+u_{4}^{5}-5\left(u_{1} u_{4}-u_{2} u_{3}\right)\left(u_{1}^{2} u_{3}-u_{2}^{2} u_{1}-u_{3}^{2} u_{4}+u_{4}^{2} u_{2}\right)=-F .
\end{gathered}
$$

These coefficients were essentially given by Ramanujan in his first notebook [39], see Berndt [1, Part IV, p. 38]. They also occur in [43].

We next introduce two additional unknowns, $\theta$ and $T$, defined by the equations

$$
\begin{gathered}
u_{1} u_{4}-u_{2} u_{3}=2 \theta, \\
u_{1}^{2} u_{3}+u_{4}^{2} u_{1}-u_{2}^{2} u_{1}-u_{3}^{2} u_{4}=2 T,
\end{gathered}
$$


in which a kind of skew symmetry will be noticed. The natural procedure is now to determine $u_{1}, u_{2}, u_{3}, u_{4}$ in terms of $\theta, T$ and the coefficients of the reduced quintic by using equations (1), (2), (5) and (6) only. When this has been done, substitute the results in (3) and (4), and we have reached the penultimate stage of our journey by being confronted with two simultaneous equations in the unknowns $\theta$ and $T$.

¿From (1) and (5) we have

$$
u_{1} u_{4}=-C+\theta, \quad u_{2} u_{3}=-C-\theta,
$$

while from (2) and (6) we have

$$
u_{1}^{2} u_{3}+u_{4}^{2} u_{2}=-D+T, \quad u_{2}^{2} u_{1}+u_{3}^{2} u_{4}=-D-T ;
$$

and hence it follows that

$$
\begin{aligned}
& u_{1}^{2} u_{3}-u_{4}^{2} u_{2}= \pm \sqrt{(D-T)^{2}+4(C-\theta)^{2}(C+\theta)}=: R_{1}, \text { say } \\
& u_{2}^{2} u_{1}-u_{3}^{2} u_{4}= \pm \sqrt{(D+T)^{2}+4(C+\theta)^{2}(C-\theta)}=: R_{2}, \text { say. }
\end{aligned}
$$

Watson makes use of the identities

$$
\begin{aligned}
& \left(u_{1}^{2} u_{3}-u_{4}^{2} u_{2}\right)^{2}=\left(u_{1}^{2} u_{3}+u_{4}^{2} u_{2}\right)^{2}-4\left(u_{1} u_{4}\right)^{2}\left(u_{2} u_{3}\right), \\
& \left(u_{2}^{2} u_{1}-u_{3}^{2} u_{4}\right)^{2}=\left(u_{2}^{2} u_{1}+u_{3}^{2} u_{4}\right)^{2}-4\left(u_{2} u_{3}\right)^{2}\left(u_{1} u_{4}\right) .
\end{aligned}
$$

These last equations enable us to obtain simple expressions for the various combinations of the $u^{\prime} s$ which occur in (3) and (4). Thus, in respect of (3), we have

$$
u_{1}^{3} u_{2}=\frac{u_{1}^{2} u_{3} \cdot u_{1} u_{2}^{2}}{u_{2} u_{3}}
$$

with similar expressions for $u_{2}^{3} u_{4}, u_{3}^{3} u_{1}, u_{4}^{3} u_{3}$. When we substitute these values in (3) and perform some quite straightforward reductions, we obtain the equation

$$
C\left(D^{2}-T^{2}\right)+\left(C^{2}-\theta^{2}\right)\left(C^{2}+3 \theta^{2}-E\right)=R_{1} R_{2} \theta .
$$

This shows incidentally that, when $\theta$ and $T$ have been determined, the signs of $R_{1}$ and $R_{2}$ cannot be assumed arbitrarily but have to be selected so that $R_{1} R_{2}$ has a uniquely determinate value. The effect of changing the signs of both $R_{1}$ and $R_{2}$ is merely to interchange $u_{1}$ with $u_{4}$ and $u_{2}$ with $u_{3}$.

The result of rationalising (7) by squaring is the more formidable equation

$$
\begin{gathered}
\left(D^{2}-T^{2}\right)^{2}+2 C\left(D^{2}-T^{2}\right)\left(C^{2}+3 \theta^{2}\right)-8 C \theta^{2}\left(D^{2}+T^{2}\right) \\
+\left(C^{2}-\theta^{2}\right)\left(C^{2}-5 \theta^{2}\right)^{2}+16 D \theta^{3} T+E^{2}\left(C^{2}-\theta^{2}\right) \\
-2 C E\left(D^{2}-T^{2}\right)-2 E\left(C^{2}-\theta^{2}\right)\left(C^{2}+3 \theta^{2}\right)=0 .
\end{gathered}
$$


This disposes of (3) for the time being, and we turn to (4). The formulae which now serve our purpose are

$$
u_{1}^{5}=\frac{\left(u_{1}^{2} u_{3}\right)^{2}\left(u_{2}^{2} u_{1}\right)}{\left(u_{2} u_{3}\right)^{2}}, \text { etc., }
$$

with three similar formulae. When these results are inserted in (4) and the equation so obtained is simplified as much as possible, we have an equation which I do not propose to write down, because it would be a little tedious; it has a sort of family resemblance to (7) in that it is of about the same degree of complexity and it involves the unknowns $\theta$ and $T$ and the product $R_{1} R_{2}$ rationally.

MAPLE gives the equation as

$$
\begin{gathered}
\left(D^{2}-T^{2}\right)\left(D \theta^{2}+2 C T \theta+C^{2} D\right)+2\left(C^{2}-\theta^{2}\right)\left(3 C D \theta^{2}-T \theta^{3}\right) \\
-R_{1} R_{2}\left(T \theta^{2}+2 C D \theta+C^{2} T\right)+\left(C^{2}-\theta^{2}\right)^{2}(20 T \theta-F)=0 .
\end{gathered}
$$

When we substitute for this product $R_{1} R_{2}$ the value which is supplied by (7), we obtain an equation which is worth writing out in full, namely

$$
\begin{gathered}
(D \theta+C T)\left(D^{2}-T^{2}\right)+T\left(C^{2}-5 \theta^{2}\right)^{2}-2 C D E \theta \\
-E T\left(C^{2}+\theta^{2}\right)+F \theta\left(C^{2}-\theta^{2}\right)=0 .
\end{gathered}
$$

We now have two simultaneous equations, (8) and (9), in which the only unknowns are $\theta$ and $T$. When these equations have been solved, the values of $u_{1}, u_{2}, u_{3}, u_{4}$ are immediately obtainable from formulae of the type giving $u_{1}^{5}$ in the form of fifth roots, and our quest will have reached its end.

Watson means that $u_{1}$ can be given as a fifth root of an expression involving the coefficients of the quintic, $R_{1}$ and $R_{2}$.

An inspection of this pair of equations, however, suggests that we may still have a formidable task in front of us.

It has to be admitted that, to all intents and purposes, this task is shirked by Young. In place of (8) and (9), the equations to which his analysis leads him are modified forms of (8) and (9). They are obtainable from (8) and (9) by taking new unknowns in place of $\theta$ and $T$, the new unknowns $t$ and $\psi$ being given in terms of our unknowns by the formulae

$$
T=\theta t, \quad \theta^{2}=\psi
$$

Young's simultaneous equations are cubic-quartic and quadratic-cubic respectively in $\psi$ and $t$. When the original quintic equation is Abelian, they possess a 
rational set of solutions.

Young's pair of simultaneous equations for $t$ and $\psi$ are

$$
\begin{gathered}
\left(D^{2}-\psi t^{2}\right)^{2}+2 C\left(D^{2}-\psi t^{2}\right)\left(C^{2}+3 \psi\right)-8 C \psi\left(D^{2}+\psi t^{2}\right)+\left(C^{2}-\psi\right)\left(C^{2}-5 \psi\right)^{2} \\
+16 D \psi^{2} t+E^{2}\left(C^{2}-\psi\right)-2 C E\left(D^{2}-\psi t^{2}\right)-2 E\left(C^{2}-\psi\right)\left(C^{2}+3 \psi\right)=0
\end{gathered}
$$

and

$$
(D+C t)\left(D^{2}-\psi t^{2}\right)+t\left(C^{2}-5 \psi\right)^{2}-2 C D E-E t\left(C^{2}+\psi\right)+F\left(C^{2}-\psi\right)=0 .
$$

Young goes on to suggest that, in numerical examples, his pair of simultaneous equations should be solved by inspection. He does, in fact, solve the equations by inspection in each of the numerical examples that he considers, and, although he says it is possible to eliminate either of the unknowns in order to obtain a single equation in the other unknown, he does not work out the eliminant. You will probably realize that the solution by inspection of a pair of simultaneous equations of so high a degree is likely to be an extremely tedious task, and you will not be mistaken in your assumption. Consequently Young's investigations have not got the air of finality about them which could have been desired.

Fortunately, however, the end of the story is implicitly told in the paper by Cayley on the sextic resolvent which I have already described to you and which had been published over a quarter of a century earlier. It is, in fact, easy to establish the relations

$$
z_{1} z_{2}+\cdots-z_{1} z_{3}-\cdots=a^{2}\left(x_{1} x_{2}+\cdots-x_{1} x_{3}-\cdots\right)=a^{2} \phi_{1},
$$

and also to prove that the expression on the left is equal to

$$
5\left(u_{1} u_{4}-u_{2} u_{3}\right) \sqrt{5}
$$

so that

$$
10 \theta \sqrt{5}=a^{2} \phi_{1}
$$

Watson is using the relation $z_{i}=a x_{i}+b(i \in\{1,2,3,4,5\})$ to obtain the first equality.

With $z_{r}=\omega^{r} u_{1}+\omega^{2 r} u_{2}+\omega^{3 r} u_{3}+\omega^{4 r} u_{4} \quad(r \in\{1,2,3,4,5\})$ MAPLE gives

$$
z_{1} z_{2}+\cdots-z_{1} z_{3}-\cdots=5\left(u_{1} u_{4}-u_{2} u_{3}\right)\left(\omega-\omega^{2}-\omega^{3}+\omega^{4}\right)
$$

so that

$$
z_{1} z_{2}+\cdots-z_{1} z_{3}-\cdots=5\left(u_{1} u_{4}-u_{2} u_{3}\right) \sqrt{5}
$$


since

$$
\omega-\omega^{2}-\omega^{3}+\omega^{4}=\sqrt{5}
$$

Consequently, to obtain a value of $\theta$ which satisfies Young's simultaneous equations, all that is necessary is to obtain a root of Cayley's sextic resolvent; and the determination of a rational value of $\phi^{2}$ which satisfies Cayley's sextic resolvent is a perfectly straightforward process, since any such value of $a^{2} \phi^{2}$ must be an integer which is a factor of $1600000000 M^{2}$ when the coefficients in the standard form of the quintic are integers, and so the number of trials which have to be made to ascertain the root is definitely limited.

The quantity $M$ is defined on Watson's sheet 1 . The constant term of Cayley's sextic resolvent is $40000 M$.

When $\theta$ has been thus determined, Young's pair of equations contain one unknown $T$ only, and there is no difficulty at all in finding the single value of $T$ which satisfies both of them by a series of trials exactly resembling the set of trials by which $\theta$ was determined.

\section{WATSON'S METHOD OF FINDING A REAL ROOT OF THE SOLVABLE QUINTIC EQUATION $a x^{5}+5 b x^{4}+10 c x^{3}+10 d x^{2}+5 e x+f=0$}

First transform the quintic into reduced form

$$
x^{5}+10 C x^{3}+10 D x^{2}+5 E x+F=0 .
$$

Watson's step by step procedure gives a real root of the reduced equation in the form $x=u_{1}+u_{2}+u_{3}+u_{4}$. The other four roots of the equation have the form $\omega^{j} u_{1}+\omega^{2 j} u_{2}+\omega^{3 j} u_{3}+\omega^{4 j} u_{4}(j=1,2,3,4)$, where $\omega=\exp (2 \pi i / 5)$.

INPUT: $C, D, E, F$

Step 1. Find a positive integer $k$ such that

$$
k \mid 16 \times 10^{8} \times M^{2}, \quad \epsilon \sqrt{k} / a \text { is a root of }(0) \text { for } \epsilon=1 \text { or }-1 .
$$

Step 2. Determine $\theta$ from

$$
\theta=\frac{\epsilon a \sqrt{k}}{10 \sqrt{5}}
$$

Step 3. Put the value of $\theta$ into (7) and (9) and then add and 
subtract multiples of these equations as necessary to determine $T$.

Step 4. Determine $R_{1}$ from

$$
R_{1}=\sqrt{(D-T)^{2}+4(C-\theta)^{2}(C+\theta)} .
$$

Step 5. Determine $R_{2}$ from

$$
R_{1} R_{2}=\left(C\left(D^{2}-T^{2}\right)+\left(C^{2}-\theta^{2}\right)\left(C^{2}+3 \theta^{2}-E\right)\right) / \theta .
$$

Step 6. Determine $u_{1}$ from

$$
u_{1}=\left(\frac{X^{2} Y}{Z^{2}}\right)^{1 / 5}
$$

where

$$
X=\left(-D+T+R_{1}\right) / 2, \quad Y=\left(-D-T+R_{2}\right) / 2, \quad Z=-C-\theta .
$$

Step 7. Determine $u_{4}$ from

$$
u_{1} u_{4}=-C+\theta .
$$

Step 8. Determine $u_{2}$ from

$$
u_{4}^{2} u_{2}=\left(-D+T-R_{1}\right) / 2 .
$$

Step 9. Determine $u_{3}$ from

$$
u_{2} u_{3}=-C-\theta .
$$

OUTPUT: A real root of the quintic is $x=u_{1}+u_{2}+u_{3}+u_{4}$.

The process which I have now described of solving an Abelian quintic by making use of the work of both Cayley and Young is a perfectly practical one, and, as I have already implied, I have used it to solve rather more than 100 Abelian quintics. If any of you would like to attempt the solution of an Abelian quintic, you will find enough information about Ramanujan's quintic given at the foot of the third sheet to enable you to complete the solution. You may remember that I mentioned that the equation was connected with the elliptic functions for which the period-quotient was $\sqrt{-79}$, and you will see the number 79 appearing somewhat unobtrusively in the values which I have quoted for the $u$ 's.

This is the end of Watson's lecture. We have made a few corrections to the text: for example, in one place Watson wrote "cubic" when he clearly meant "quintic." Below are the three handout sheets that he refers to in his lecture. 
24 AN UNPUBLISHED LECTURE OF WATSON ON SOLVING THE QUINTIC

\section{SHEET 1}

The denumerate form of the quintic equation is

$$
p_{0} y^{5}+p_{1} y^{4}+p_{2} y^{3}+p_{3} y^{2}+p_{4} y+p_{5}=0
$$

The standard form of the quintic equation is

$$
a x^{5}+5 b x^{4}+10 c x^{3}+10 d x^{2}+5 e x+f=0 . \quad(x=10 y)
$$

The reduced form of the quintic equation is

$$
z^{5}+10 C z^{3}+10 D z^{2}+5 E z+F=0 . \quad(z=a x+b)
$$

The sextic resolvent is

$$
a^{6} \phi^{6}-100 K a^{4} \phi^{4}+2000 L a^{2} \phi^{2}-800 a^{2} \phi \sqrt{5 \Delta}+40000 M=0
$$

where

$$
\begin{aligned}
K= & a e-4 b d+3 c^{2} \\
L= & -2 a^{2} d f+3 a^{2} e^{2}+6 a b c f-14 a b d e-2 a c^{2} e+8 a c d^{2}-4 b^{3} f \\
& +10 b^{2} c e+20 b^{2} d^{2}-40 b c^{2} d+15 c^{4} \\
M= & a^{3} c f^{2}-2 a^{3} d e f+a^{3} e^{3}-a^{2} b^{2} f^{2}-4 a^{2} b c e f+8 a^{2} b d^{2} f-2 a^{2} b d e^{2} \\
& -2 a^{2} c^{2} d f-11 a^{2} c^{2} e^{2}+28 a^{2} c d^{2} e-16 a^{2} d^{4}+6 a b^{3} e f \\
& -12 a b^{2} c d f+35 a b^{2} c e^{2}-40 a b^{2} d^{2} e+6 a b c^{3} f-70 a b c^{2} d e \\
& +80 a b c d^{3}+35 a c^{4} e-40 a c^{3} d^{2}-25 b^{4} e^{2}+100 b^{3} c d e \\
& -50 b^{2} c^{3} e-100 b^{2} c^{2} d^{2}+100 b c^{4} d-25 c^{6} .
\end{aligned}
$$

The discriminant $\Delta$ of the quintic equation in its standard form is equal to the product of the squared differences of the roots multiplied by $a^{8} / 3125$. The value of the discriminant $\Delta$ in terms of the coefficients is 


$$
\begin{aligned}
& a^{4} f^{4}-20 a^{3} b c f^{3}-120 a^{3} c d f^{3}+160 a^{3} c e^{2} f^{2}+360 a^{3} d^{2} e f^{2} \\
& -640 a^{3} d e^{3} f+256 a^{3} e^{5}+160 a^{2} b^{2} d f^{3}-10 a^{2} b^{2} e^{2} f^{2} \\
& +360 a^{2} b c^{2} f^{3}-1640 a^{2} b c d e f^{2}+320 a^{2} b c e^{3} f-1440 a^{2} b d^{3} f^{2} \\
& +4080 a^{2} b d^{2} c^{2} f-1920 a^{2} b d e^{4}-1440 a^{2} c^{3} e f^{2}+2640 a^{2} c^{2} d^{2} f^{2} \\
& +4480 a^{2} c^{2} d e^{2} f-2560 a^{2} c^{2} e^{4}-10080 a^{2} c d^{3} e f+5760 a^{2} c d^{2} e^{3} \\
& +3456 a^{2} d^{5} f-2160 a^{2} d^{4} e^{2}-640 a b^{3} c f^{3}+320 a b^{3} d e f^{2} \\
& -180 a b^{3} c^{3} f+4080 a b^{2} c^{2} e f^{2}+4480 a b^{2} c d^{2} f^{2}-14920 a b^{2} c d e^{2} f \\
& +7200 a b^{2} c e^{4}+960 a b^{2} d^{3} e f-600 a b^{2} d^{2} e^{3}-10080 a b c^{3} d f^{2} \\
& +960 a b c^{3} e^{2} f+28480 a b c^{2} d^{2} e f-16000 a b c^{2} d e^{3}-11520 a b c d^{4} f \\
& +7200 a b c d^{3} e^{2}+3456 a c^{5} f^{2}-11520 a c^{4} d e f+6400 a c^{4} e^{3} \\
& +5120 a c^{3} d^{3} f-3200 a c^{3} d^{2} e^{2}+256 b^{5} f^{3}-1920 b^{4} c e f^{2} \\
& -2560 b^{4} d^{2} f^{2}+7200 b^{4} d e^{2} f-3375 b^{4} c^{4}+5760 b^{3} c^{2} d f^{2} \\
& -600 b^{3} c^{2} e^{2} f-16000 b^{3} c d^{2} e f+9000 b^{3} c d e^{3}+6400 b^{3} d^{4} f \\
& -4000 b^{3} d^{3} e^{2}-2160 b^{2} c^{4} f^{2}+7200 b^{2} c^{3} d e f-4000 b^{2} c^{3} e^{3} \\
& -3200 b^{2} c^{2} d^{3} f+2000 b^{2} c^{2} d^{2} e^{2} .
\end{aligned}
$$

\section{SHEET 2}

(Place pentagram diagram here)

\section{SHEET 3}

The roots of the quintic in its reduced form are

$$
z_{r+1}=\omega^{r} u_{1}+\omega^{2 r} u_{2}+\omega^{3 r} u_{3}+\omega^{4 r} u_{4}
$$

with $\omega=\exp (2 \pi i / 5), \quad r=1,2,3,4,0$.

$$
\begin{aligned}
& u_{1} u_{4}+u_{2} u_{3}=-2 C . \\
& u_{1}^{2} u_{3}+u_{2}^{2} u_{1}+u_{3}^{2} u_{4}+u_{4}^{2} u_{2}=-2 D . \\
& u_{1}^{2} u_{4}^{2}+u_{2}^{2} u_{3}^{2}-u_{1} u_{2} u_{3} u_{4}-u_{1}^{3} u_{2}-u_{2}^{3} u_{4}-u_{3}^{3} u_{1}-u_{4}^{3} u_{3}=E . \\
& u_{1}^{5}+u_{2}^{5}+u_{3}^{5}+u_{4}^{5}-5\left(u_{1} u_{4}-u_{2} u_{3}\right)\left(u_{1}^{2} u_{3}-u_{2}^{2} u_{1}-u_{3}^{2} u_{4}+u_{4}^{2} u_{2}\right)=-F .
\end{aligned}
$$


26 AN UNPUBLISHED LECTURE OF WATSON ON SOLVING THE QUINTIC

New unknowns, $\theta$ and $T$, defined by

$$
\begin{aligned}
& u_{1} u_{4}-u_{2} u_{3}=2 \theta, \\
& u_{1}^{2} u_{3}+u_{4}^{2} u_{2}-u_{2}^{2} u_{1}-u_{3}^{2} u_{4}=2 T . \\
& u_{1} u_{4}=-C+\theta, \quad u_{2} u_{3}=-C-\theta . \\
& u_{1}^{2} u_{3}+u_{4}^{2} u_{2}=-D+T, \quad u_{2}^{2} u_{1}+u_{3}^{2} u_{4}=-D-T . \\
& u_{1}^{2} u_{3}-u_{4}^{2} u_{2}= \pm \sqrt{(D-T)^{2}+4(C-\theta)^{2}(C+\theta)}=R_{1}, \\
& u_{2}^{2} u_{1}-u_{3}^{2} u_{4}= \pm \sqrt{(D+T)^{2}+4(C+\theta)^{2}(C-\theta)}=R_{2} . \\
& u_{1}^{3} u_{2}=\left(u_{1}^{2} u_{3}\right)\left(u_{2}^{2} u_{1}\right) /\left(u_{2} u_{3}\right), \text { etc. }, \quad u_{1}^{5}=\left(u_{1}^{2} u_{3}\right)^{2}\left(u_{2}^{2} u_{1}\right) /\left(u_{2} u_{3}\right)^{2}, \text { etc. } \\
& C\left(D^{2}-T^{2}\right)+\left(C^{2}-\theta^{2}\right)\left(C^{2}+3 \theta^{2}-E\right)=R_{1} R_{2} \theta . \\
& \left(D^{2}-T^{2}\right)^{2}+2 C\left(D^{2}-T^{2}\right)\left(C^{2}+3 \theta^{2}\right)-8 C \theta^{2}\left(D^{2}+T^{2}\right) \\
& +\left(C^{2}-\theta^{2}\right)^{2}\left(C^{2}-5 \theta^{2}\right)^{2}+16 D \theta^{3} T+E^{2}\left(C^{2}-\theta^{2}\right) \\
& -2 C E\left(D^{2}-T^{2}\right)-2 E\left(C^{2}-\theta^{2}\right)\left(C^{2}+3 \theta^{2}\right)=0 . \\
& (D \theta+C T)\left(D^{2}-T^{2}\right)+T\left(C^{2}-5 \theta^{2}\right)^{2}-2 C D E \theta \\
& -E T\left(C^{2}+\theta^{2}\right)+F \theta\left(C^{2}-\theta^{2}\right)=0 .
\end{aligned}
$$

Young's substitutions are

$$
T=\theta t, \quad \theta^{2}=\psi
$$

The connexion between the $\theta$ above and the $\phi$ of Cayley's sextic resolvent is

$$
10 \theta \sqrt{5}=a^{2} \phi
$$

The denumerate quintic of Ramanujan's problem is

$$
y^{5}-y^{4}+y^{3}-2 y^{2}+3 y-1=0 .
$$

For this quintic, $C=6, D=-156, E=4592, F=-47328$.

$$
\begin{gathered}
z=10 y-2, \quad \theta=-10 \sqrt{5}, \quad t=-10, \quad T=100 \sqrt{5} . \\
u_{1}^{5}, \quad u_{4}^{5}=-13168-6400 \sqrt{5} \pm(2160+960 \sqrt{5}) \sqrt{79(5-2 \sqrt{5})},
\end{gathered}
$$




$$
\begin{gathered}
u_{2}^{5}, \quad u_{3}^{5}=-13168+6400 \sqrt{5} \pm(2160-960 \sqrt{5}) \sqrt{79(5+2 \sqrt{5})} . \\
R_{1}^{2}, \quad R_{2}^{2}=79(800 \pm 160 \sqrt{5}), \quad R_{1} R_{2}=-320 \times 79 \sqrt{5} .
\end{gathered}
$$

We remark that Young's equations for $t$ and $\psi$ in this example are:

$$
\begin{gathered}
\left(24336-\psi t^{2}\right)+12\left(24336-\psi t^{2}\right)(36+3 \psi)-48 \psi\left(24336+\psi t^{2}\right) \\
+(36-\psi)(36-5 \psi)^{2}-2496 \psi^{2} t-581898240-21086464 \psi+55104 \psi t^{2} \\
-9184(36-\psi)(36+3 \psi)=0
\end{gathered}
$$

and

$$
(-156+6 t)\left(24336-\psi t^{2}\right)+t(36-5 \psi)^{2}+6892416-4592 t(36+\psi)+47328 \psi=0,
$$

so that $t=-10$ and $\psi=500$ in agreement with Watson.

We conclude by giving three examples illustrating Watson's procedure.

Example 1. $x^{5}-5 x+12=0$

The Galois group of $x^{5}-5 x+12$ is $D_{5}$. Here

$$
\begin{gathered}
a=1, b=0, c=0, d=0, e=-1, f=12, \\
C=0, D=0, E=-1, F=12, \\
K=-1, L=3, M=-1, \\
\Delta=5 \times 2^{12}, \sqrt{5 \Delta}=520 .
\end{gathered}
$$

Equation (0) is

$$
\phi^{6}+100 \phi^{4}+6000 \phi^{2}-256000 \phi-40000=0 .
$$

Step 1

$$
k=10 \text {. }
$$

Step 2

$$
\theta=\frac{1}{\sqrt{5}} .
$$

Step 3

$$
T=\frac{2}{\sqrt{5}}
$$


Step 4

$$
R_{1}=\frac{2}{5} \sqrt{5+\sqrt{5}}
$$

Step 5

$$
R_{2}=-\frac{2}{5} \sqrt{5-\sqrt{5}}
$$

Step 6

$$
\begin{gathered}
X=\frac{\sqrt{5}+\sqrt{5+\sqrt{5}}}{5}, \quad Y=\frac{-\sqrt{5}-\sqrt{5-\sqrt{5}}}{5}, \quad Z=-\frac{1}{\sqrt{5}} \\
u_{1}=-\left(\frac{(\sqrt{5}+\sqrt{5+\sqrt{5}})^{2}(\sqrt{5}+\sqrt{5-\sqrt{5}}}{25}\right)^{1 / 5} .
\end{gathered}
$$

Step 7

$$
u_{4}=-\left(\frac{(\sqrt{5}-\sqrt{5+\sqrt{5}})^{2}(\sqrt{5}-\sqrt{5-\sqrt{5}}}{25}\right)^{1 / 5}
$$

Step 8

$$
u_{2}=-\left(\frac{(\sqrt{5}-\sqrt{5-\sqrt{5}})^{2}(-\sqrt{5}-\sqrt{5+\sqrt{5}}}{25}\right)^{1 / 5}
$$

Step 9

$$
u_{3}=-\left(\frac{(\sqrt{5}+\sqrt{5-\sqrt{5}})^{2}(-\sqrt{5}+\sqrt{5+\sqrt{5}}}{25}\right)^{1 / 5} .
$$

This agrees with [43, Example 1].

Example 2. $x^{5}+15 x+12=0$

The Galois group of $x^{5}-5 x+12$ is $F_{20}$. Here

$$
\begin{gathered}
a=1, b=0, c=0, d=0, e=3, f=12, \\
C=0, D=0, E=3, F=12, \\
K=3, L=27, M=27, \\
\Delta=2^{10} \times 3^{4}, \sqrt{5 \Delta}=288 \sqrt{5} .
\end{gathered}
$$

Equation (0) is

$$
\phi^{6}-300 \phi^{4}+54000 \phi^{2}-230400 \sqrt{5} \phi+1080000=0 .
$$


Step 1

$$
k=180 .
$$

Step 2

$$
\theta=\frac{3}{5}
$$

Step 3

$$
T=\frac{6}{5}
$$

Step 4

$$
R_{1}=\frac{12 \sqrt{10}}{25}
$$

Step 5

$$
R_{2}=\frac{6 \sqrt{10}}{25}
$$

Step 6

$$
\begin{gathered}
X=\frac{15+6 \sqrt{10}}{25}, \quad Y=\frac{-15+3 \sqrt{10}}{25}, Z=-\frac{3}{5}, \\
u_{1}=\left(\frac{-75-21 \sqrt{10}}{125}\right)^{1 / 5} .
\end{gathered}
$$

Step 7

$$
u_{4}=\left(\frac{-75+21 \sqrt{10}}{125}\right)^{1 / 5}
$$

Step 8

$$
u_{2}=\left(\frac{225-72 \sqrt{10}}{125}\right)^{1 / 5}
$$

Step 9

$$
u_{3}=\left(\frac{225+72 \sqrt{10}}{125}\right)^{1 / 5} .
$$

This agrees with [43, Example 2].

Example 3. $x^{5}-25 x^{3}+50 x^{2}-25=0$

The Galois group of $x^{5}-25 x^{3}+50 x^{2}-25$ is $\mathbb{Z}_{5}$. Here

$$
a=1, b=0, c=-5 / 2, d=5, e=0, f=-25,
$$


30 AN UNPUBLISHED LECTURE OF WATSON ON SOLVING THE QUINTIC

$$
\begin{gathered}
C=-5 / 2, D=5, E=0, F=-25, \\
K=75 / 4, L=5375 / 16, M=-30625 / 64, \\
\Delta=5^{7} \times 7^{2}, \sqrt{5 \Delta}=5^{4} \times 7 .
\end{gathered}
$$

Equation (0) is

$$
\phi^{6}-1875 \phi^{4}+671875 \phi^{2}-3500000 \phi-19140625=0 .
$$

Step 1

$$
k=625 .
$$

Step 2

$$
\theta=\frac{-\sqrt{5}}{2}
$$

Step 3

$$
T=0 .
$$

Step 4

$$
R_{1}=\sqrt{-25+10 \sqrt{5}}
$$

Step 5

$$
R_{2}=\sqrt{-25-10 \sqrt{5}}
$$

Step 6

$$
\begin{gathered}
X=\frac{-5+\sqrt{-25+10 \sqrt{5}}}{2}, Y=\frac{-5+\sqrt{-25-10 \sqrt{5}}}{2}, Z=\frac{5+\sqrt{5}}{2}, \\
u_{1}=\left(\frac{X^{2} Y}{Z^{2}}\right)^{\frac{1}{5}}=\frac{25+15 \sqrt{5}+5 \sqrt{-130-58 \sqrt{5}}}{4} .
\end{gathered}
$$

Step 7

$$
u_{4}=\frac{25+15 \sqrt{5}-5 \sqrt{-130-58 \sqrt{5}}}{4} .
$$

Step 8

$$
u_{2}=\frac{25-15 \sqrt{5}+5 \sqrt{-130+58 \sqrt{5}}}{4} .
$$

Step 9

$$
u_{3}=\frac{25-15 \sqrt{5}-5 \sqrt{-130+58 \sqrt{5}}}{4} .
$$

\section{REFERENCES}


1. Bruce C. Berndt, Ramanujan's Notebooks, Springer-Verlag, New York, Part I (1985), Part II (1989), Part III (1991), Part IV (1994), Part V (1998).

2. Bruce C. Berndt, Heng Huat Chan, and Liang-Cheng Zhang, Ramanujan's class invariants and cubic continued fraction, Acta Arithmetica 73 (1995), 67-85.

3. Bruce C. Berndt, Heng Huat Chan, and Liang-Cheng Zhang, Ramanujan's class invariants, Kronecker's limit formula, and modular equations, Transactions of the American Mathematical Society 349 (1997), 2125-2173.

4. Bruce C. Berndt, Youn-Seo Choi, and Soon-Yi Kang, The problems submitted by Ramanujan to the Journal of the Indian Mathematical Society, in Continued Fractions: From Analytic Number Theory to Constructive Approximation, B. C. Berndt and F. Gesztesy, eds., Contemp. Math. No. 236, American Mathematical Society, Providence, RI, 1999, pp. 15-56.

5. William S. Burnside and Arthur W. Panton, The Theory of Equations, 2 vols., Dover, New York, 1960.

6. Arthur Cayley, On a new auxiliary equation in the theory of equations of the fifth order, Philosophical Transactions of the Royal Society of London CLI (1861), 263-276. [15, Vol. IV, Paper 268, pp. 309-324.]

7. Arthur Cayley, Note on Mr. Jerrard's researches on the equation of the fifth order, The London, Edinburgh and Dublin Philosophical Magazine and Journal of Science XXI (1861), 210-214. [15, Vol. V, Paper 310, pp. 50-54.]

8. Arthur Cayley, On a theorem of Abel's relating to equations of the fifth order, The London, Edinburgh and Dublin Philosophical Magazine and Journal of Science XXI (1861), 257-263. [15, Vol. V, Paper 311, pp. 55-61.]

9. Arthur Cayley, Note on the solution of an equation of the fifth order, The London, Edinburgh and Dublin Philosophical Magazine and Journal of Science XXIII (1862), pp. 195, 196. [15, Vol. V, Paper 316, p. 77.]

10. Arthur Cayley, Final remarks on Mr. Jerrard's theory of equations of the fifth order, The London, Edinburgh and Dublin Philosophical Magazine and Journal of Science XXIV (1862), 290. [15, Vol. V, Paper 321, p. 89.]

11. Arthur Cayley, Note on the solvability of equations by means of radicals, 
32 AN UNPUBLISHED LECTURE OF WATSON ON SOLVING THE QUINTIC

The London, Edinburgh and Dublin Philosophical Magazine and Journal of Science XXXVI (1868), pp. 386, 387. [15, Vol. VII, Paper 421, pp. 13-14.]

12. Arthur Cayley, On a theorem of Abel's relating to a quintic equation, Cambridge Philosophical Society Proceedings III (1880), 155-159. [15, Vol. XI, Paper 741, pp. 132-135.]

13. Arthur Cayley, A solvable case of the quintic equation, Quarterly Journal of Pure and Applied Mathematics XVIII (1882), 154-157. [15, Vol. XI, Paper 777, pp. 402-404.]

14. Arthur Cayley, On a soluble quintic equation, American Journal of Mathematics XIII (1891), 53-58. [15, Vol. XIII, Paper 914, pp. 88-92.]

15. Arthur Cayley, The Collected Mathematical Papers of Arthur Cayley, Cambridge University Press, Vol. I (1889), Vol. II (1889), Vol. III (1890), Vol. IV (1891), Vol. V (1892), Vol. VI (1893), Vol. VII (1894), Vol. VIII (1895), Vol. IX (1896), Vol. X (1896), Vol. XI (1896), Vol. XII (1897), Vol. XIII (1897), Vol. XIV (1898).

16. Heng Huat Chan, Ramanujan-Weber class invariant $G_{n}$ and Watson's empirical process, Journal of the London Mathematical Society 57 (1998), 545-561.

17. James Cockle, Researches in the higher algebra, Memoirs of the Literary and Philosophical Society of Manchester XV (1858), 131-142.

18. James Cockle, Sketch of a theory of transcendental roots, The London, Edinburgh and Dublin Philosophical Magazine and Journal of Science XX (1860), $145-148$.

19. James Cockle, On the resolution of quintics, Quarterly Journal of Pure and Applied Mathematics 4 (1861), 5-7.

20. James Cockle, Notes on the higher algebra, Quarterly Journal of Pure and Applied Mathematics 4 (1861), 49-57.

21. James Cockle, On transcendental and algebraic solution-supplementary paper, The London, Edinburgh and Dublin Philosophical Magazine and Journal of Science XXIII (1862), 135-139.

22. Winifred A. Cooke, George Neville Watson, Mathematical Gazette 49 (1965), 
$256-258$.

23. David A. Cox, Primes of the Form $x^{2}+n y^{2}$, Wiley, New York, 1989.

24. David S. Dummit, Solving solvable quintics, Mathematics of Computation 57 (1991), 387-401.

25. David S. Dummit and Richard M. Foote, Abstract Algebra, Prentice Hall, New Jersey, 1991.

26. W. L. Ferrar, Higher Algebra, Oxford University Press, Oxford, 1950.

27. Joseph A. Gallian, Contemporary Abstract Algebra, Fourth Edition, Houghton Mifflin Co., Boston MA, 1998.

28. J. C. Glashan, Notes on the quintic, American Journal of Mathematics 7 (1885), 178-179.

29. Robert Harley, On the method of symmetric products, and its application to the finite algebraic solution of equations, Memoirs of the Literary and Philosophical Society of Manchester XV (1859), 172-219.

30. Robert Harley, On the theory of quintics, Quarterly Journal of Pure and Applied Mathematics 3 (1859), 343-359.

31. Robert Harley, On the theory of the transcendental solution of algebraic equations, Quarterly Journal of Pure and Applied Mathematics 5 (1862), 337-361.

32. R. Bruce King, Beyond the Quartic Equation, Birkhäuser, Boston, 1996.

33. Sigeru Kobayashi and Hiroshi Nakagawa, Resolution of solvable quintic equation, Mathematica Japonicae 37 (1992), 883-886.

34. John Emory McClintock, On the resolution of equations of the fifth degree, American Journal of Mathematics 6 (1883-1884), 301-315.

35. John Emory McClintock, Analysis of quintic equations, American Journal of Mathematics 8 (1885), 45-84. 
34 AN UNPUBLISHED LECTURE OF WATSON ON SOLVING THE QUINTIC

36. John Emory McClintock, Further researches in the theory of quintic equations, American Journal of Mathematics 20 (1898), 157-192.

37. Srinivasa Ramanujan, Modular equations and approximations to $\pi$, Quarterly Journal of Mathematics 45 (1914), 350-372. [40: pp. 23-39.]

38. Srinivasa Ramanujan, Question 699, Journal of the Indian Mathematical Society 7 (1917), 199. [40: p. 331.]

39. Srinivasa Ramanujan, Notebooks, 2 vols., Tata Institute of Fundamental Research, Bombay, 1957.

40. Srinivasa Ramanujan, Collected Papers of Srinivasa Ramanujan, AMS Chelsea, Providence RI, 2000.

41. Robert A. Rankin, George Neville Watson, Journal of the London Mathematical Society 41 (1966), 551-565.

42. R. Russell, On modular equations, Proceedings of the London Mathemaical Society 21 (1889-1890), 351-395.

43. Blair K. Spearman and Kenneth S. Williams, Characterization of solvable quintics $x^{5}+a x+b$, American Mathematical Monthly 101 (1994), 986-992.

44. Blair K. Spearman and Kenneth S. Williams, Demoivre's quintic and a theorem of Galois, Far East Journal of Mathematical Sciences 1 (1999), 137-143.

45. Blair K. Spearman and Kenneth S. Williams, Dihedral quintic polynomials and a theorem of Galois, Indian Journal of Pure and Applied Mathematics 30 (1999), 839-845.

46. Blair K. Spearman and Kenneth S. Williams, Conditions for the insolvabiity of the quintic equation $x^{5}+a x+b=0$, Far East Journal of Mathematical Sciences $\mathbf{3}$ (2001), 209-225.

47. Blair K. Spearman and Kenneth S. Williams, Note on a paper of Kobayashi and Nakagawa, Scientiae Mathematicae Japonicae 53 (2001), 323-334.

48. K. L. Wardle, George Neville Watson, Mathematical Gazette 49 (1965), 253-256. 
49. George N. Watson, Solution to Question 699, Journal of the Indian Mathematical Society 18 (1929-1930), 273-275.

50. George N. Watson, Theorems stated by Ramanujan (XIV): a singular modulus, Journal of the London Mathematical Society 6 (1931), 126-132.

51. George N. Watson, Some singular moduli (I), Quarterly Journal of Mathematics 3 (1932), 81-98.

52. George N. Watson, Some singular moduli (II), Quarterly Journal of Mathematics 3 (1932), 189-212.

53. George N. Watson, Singular moduli (3), Proceedings of the London Mathematical Society 40 (1936), 83-142.

54. George N. Watson, Singular moduli (4), Acta Arithmetica 1 (1936), 284-323.

55. George N. Watson, Singular moduli (5), Proceedings of the London Mathematical Society 42 (1937), 377-397.

56. George N. Watson, Singular moduli (6), Proceedings of the London Mathematical Society 42 (1937), 398-409.

57. Heinrich Weber, Lehrbuch der Algebra, 3 vols., Chelsea, New York, 1961.

58. George P. Young, Resolution of solvable equations of the fifth degree, American Journal of Mathematics 6 (1883-1884), 103-114.

59. George P. Young, Solution of solvable irreducible quintic equations, without the the aid of a resolvent sextic, American Journal of Mathematics 7 (1885), 170-177.

60. George P. Young, Solvable quintic equations with commensurable coefficients, American Journal of Mathematics 10 (1888), 99-130.

61. Liang-Cheng Zhang, Ramanujan's class invariants, Kronecker's limit formula and modular equations (II), in Analytic Number Theory: Proceedings of a Conference in Honor of Heini Halberstam, Vol. 2, B. C. Berndt, H. G. Diamond and A. J. Hildebrand, eds., Birkhäuser, Boston, 1996, pp. 817-838. 
36 AN UNPUBLISHED LECTURE OF WATSON ON SOLVING THE QUINTIC

62. Liang-Cheng Zhang, Ramanujan's class invariants, Kronecker's limit formula and modular equations (III), Acta Arithmetica 82 (1997), 379-392. 